\title{
BILATERAL ELECTROCONVULSIVE THERAPY FOR POST-TRAUMATIC STRESS DISORDER COMORBID TO DEPRESSION
}

\author{
Erhan Kavakbasi ${ }^{1}$ (D), Goktug Mert Ciftci $^{1}$ (D), Bernhard T. Baune ${ }^{1,2}$ (D)
}

${ }^{1}$ Department of Psychiatry, University Hospital Münster, Münster, GERMANY

${ }^{2}$ Department of Psychiatry, Melbourne Medical School, University of Melbourne, Melbourne, AUSTRALIA

\begin{abstract}
Aims: We aim to present a patient who was suffering from treatment-resistant depression along with psychotic features and comorbid post-traumatic stress disorder and was treated by bilateral electroconvulsive therapy. Case Report: A 58-year-old female patient was transferred to the University Hospital Münster with the diagnosis of treatment-refractory depression with psychotic features and post-traumatic stress disorder. The patient was non-responsive to unilateral electroconvulsive therapy and multiple antidepressant agents during several inpatient treatments. After initiating bilateral electroconvulsive therapy, the patient's symptoms improved significantly. Conclusion: After ruling out conventional treatment algorithms for psychotic depression comorbid to post-traumatic stress disorder, physicians can consider bilateral electroconvulsive therapy to treat complicated cases. Keywords: Stress disorders, post-traumatic, electroconvulsive therapy, depressive disorder, psychotic disorders
\end{abstract}

\section{INTRODUCTION}

Treatment-resistant depression with auditory hallucinations and comorbid post-traumatic stress disorder (PTSD) is a diagnostic and therapeutic challenge in which the role of electroconvulsive therapy (ECT) is unclear (1). A systematic review concluded that, because of the small number of cases, it remains unclear if favorable outcomes are conditioned by the improvement of depression symptoms without beneficial effects on core PTSD symptoms (1). We report the case of a 58-year-old female patient with treatment-refractory depression and PTSD, who responded neither to unilateral ECT nor to multiple antidepressant agents during several inpatient treatments. However, she achieved remission after bilateral ECT.

\section{CASE REPORT}

The present episode of depression began two years ago and was accompanied by psychotic features. The key symptoms were severely depressed mood, anhedonia, psychomotor agitation, suicidal ideations, and concentration difficulties. The patient heard the suicide-commanding and insulting voices of her father, who had abused her during her childhood. This put her under so much pressure that she only trembled and could no longer hold an orderly conversation. In addition, she suffered from flashbacks and intrusions.

Before her admission to our hospital, it had not been possible to distinguish the patients' condition between depression with psychotic symptoms and PTSD with psychosis-related experience, because the voices referred almost exclusively to previous traumatic events. She had been hospitalized for psychiatric treatment multiple times for several months during the same episode without achieving remission. Therefore, she was transferred to our university hospital.
Due to treatment resistance, we started a series of bitemporal ECT. Before ECT, the patient showed a high score in Beck's Depression Inventory-II (BDI-II: 52) and had severe PTSD symptoms, showing a score of 7 in Clinical Global Impressions - Severity Scale. The patient showed a rapid response with clinical improvements after the second ECT session. After the 7th ECT, a remission of the depressive episode was recorded (BDI-II: 8). Symptoms of PTSD had also significantly improved, having a score of 1 on the Clinical Global Impressions - Improvement Scale. The auditory hallucinations, as well as flashbacks and intrusions, disappeared completely. As a side effect of ECT, dizziness and slight memory deficits occurred, but these only lasted for a few hours. Maintenance ECT sessions at ever-increasing intervals were planned.

\section{DISCUSSION}

With major depression, comorbid PTSD, and auditory hallucinations, ECT may be an adequate therapeutic approach. Bilateral ECT was shown to be as similarly effective as high dose right unilateral ECT with acute depression, whereas low-to-moderate dose right unilateral ECT was found to be non-superior to sham treatment (2). Our case shows that ECT should not be considered ineffective before the bitemporal stimulation technique is used. Patients who have not responded to unilateral stimulation may still respond very well to bitemporal stimulation.

Acoustic hallucinations related to past maltreatment can be difficult to classify when there is PTSD comorbid to severe depression. Only a few studies show the effectiveness of ECT in improving the symptoms and reducing the suicide rate in patients with severe depression and comorbid PTSD $(3,4)$. Our case shows that ECT should be considered as a therapeutic option in these patients and can be very effective, even after no response to unilateral stimula- 
tion. Our patient had no memory loss regarding the maltreatment in her childhood, which was discussed as the mechanism of action of ECT in these patients (5). Prospective, randomized, and controlled trials are needed to determine the effectiveness of ECT in these patients.

\section{Technical Parameters of ECT Device}

ECT was performed with THYMATRON ${ }^{\circledR}$ SYSTEM IV. Thiopental was used as an anesthetic agent for ECT. An age-based dosing method was used.

Ethics Committee Approval: N/A

Informed Consent: Informed verbal consent was obtained from the patient. Conflict of Interest: The authors declare no conflict of interest.

Author Contributions: Concept: BTB, EK, GMC. Design: BTB, EK, GMC. Supervision: BTB, EK. Resources: BTB. Materials: BTB, EK, GMC. Data collection and/or Processing: EK. Analysis and/or Interpretation: BTB, EK, GMC. Literature Search: EK, BTB. Writing Manuscript: EK, GMC. Critical Review: EK, BTB, GMC.

Financial Disclosure: The authors declared that this study received no financial support.
Editor-in-Chief's Note: One of the authors of this article, Goktug Mert Ciftci, is a member of the editorial board of Turkish Medical Student Journal. However, he did not take place at any stage of the editorial decision of the manuscript. The editors who evaluated this manuscript are from other institutions.

\section{REFERENCES}

1. Youssef NA, McCall WV, Andrade C. The role of ECT in post-traumatic stress disorder: systematic review. Ann Clin Psychiatry 2017;29(1):62-70.

2. Mutz J, Vipulananthan V, Carter B et al. Comparative efficacy and acceptability of non-surgical brain stimulation for the acute treatment of major depressive episodes in adults: systematic review and network meta-analysis. BMJ 2019;364:11079.

3. Ahmadi N, Moss L, Simon E et al. Efficiacy and long-term clinical outcome of comorbid post-traumatic stress disorder and major depressive disorder after electroconvulsive therapy. Depress Anxiety 2016;33(7):640-7.

4. Kaster TS, Goldbloom DS, Daskalakis ZJ et al. Electroconvulsive therapy for depression with comorbid borderline personality disorder or post-traumatic stress disorder: a matched retrospective cohort study. Brain Stimul 2018;11(1):204-12.

5. Kellner CH, Romanella SM. ECT as a novel treatment for PTSD. J ECT 2019;35(2):e13. 